\title{
Lepton-hadronic processes and high-energy neutrinos in NGC 1275
}

\author{
N. Fraija, ${ }^{1} \dagger$ A. Marinelli, ${ }^{2}$ U. Luviano-Valenzuela,,${ }^{1,3}$ \\ A. Galván-Gaméz, ${ }^{1,3}$ and C. Peterson-Bórquez ${ }^{1,3}$ \\ ${ }^{1}$ Instituto de Astronomía, Universidad Nacional Autónoma de México, Circuito Exterior, \\ email: nifraija@astro.unam.mx \\ ${ }^{2}$ Instituto de Fisica, UNAM, Circuito Exterior \\ ${ }^{3}$ Facultad de Ciencias, UNAM, Circuito Exterior
}

\begin{abstract}
The nearby active galaxy NGC 1275, has been widely detected from radio to gamma rays. Its spectral energy distribution (SED) shows a double-peak feature, which is well explained by synchrotron self-Compton (SSC) model. However, recent TeV detections might suggest that very-high-energy $\gamma$-rays $(\mathrm{E} \geqslant 100 \mathrm{GeV})$ may not have a leptonic origin. We test a lepto-hadronic model to describe the whole SED through SSC emission and neutral pion decay resulting from $\mathrm{p} \gamma$ interactions. Also, we estimate the neutrino events expected in a $\mathrm{km}^{3}$ Cherenkov telescope.
\end{abstract}

Keywords. galaxies: individual (NGC1275), radiation mechanisms: non-thermal

\section{Introduction}

NGC 1275, also known as Perseus A and 3C 84, is the nearby active galaxy located at the center of the Perseus cluster at redshift of $z=0.0179$ (Veron (1978)). This object has been well studied in radio, optical/UV, X-ray and MeV-GeV emission (Abdo et al. 2009, Aleksić et al. 2012, Acciari et al. 2009). Recently, this source has been detected by MAGIC telescopes with a statistical significance of $6.6 \sigma$ above $100 \mathrm{GeV}$ in $46 \mathrm{hr}$ of stereo observations carried out between August 2010 and February 2011. The measured differential energy spectrum between $70 \mathrm{GeV}$ and $500 \mathrm{GeV}$ can be described by a power law with a steep spectral index of $\Gamma=-4.1 \pm 0.7_{\text {stat }} \pm 0.3_{\text {syst }}$, and the average flux above $100 \mathrm{GeV}, F_{\gamma}=1.3 \pm 0.2_{\text {stat }} \pm 0.3_{\text {syst }} \times 10^{-11} \mathrm{~cm}^{-2} \mathrm{~s}^{-1}$ (Aleksić et al. (2012)). We propose a lepto-hadronic model to describe the whole SED as the superposition of synchrotron self-Compton (SSC) emission and the neutral pion decay products.

\section{Theoretical model}

Fermi-accelerated electrons in the emitting region are described by a broken powerlaw written as: $N_{e}\left(\gamma_{e}\right) \propto \gamma_{e}^{-\alpha_{e}}$ for $\gamma_{e, m}<\gamma_{e}<\gamma_{e, b}$, and $N_{e}\left(\gamma_{e}\right) \propto \gamma_{e, b} \gamma_{e}^{-\left(\alpha_{e}+1\right)}$ for $\gamma_{e, b} \leqslant \gamma_{e}<\gamma_{e, \max }$ and co-accelerated protons at the same place for a simple power law as: $N_{p}\left(\gamma_{p}\right) \propto \gamma_{p}^{-\alpha_{p}}$, where $\alpha_{e}\left(\alpha_{p}\right)$ is the power index for electrons (protons) and $\gamma_{e, i}\left(\gamma_{p}\right)$ are the electron (proton) Lorentz factors. The indices $i, c$ and max are for minimum, break, and maximum, respectively. Assuming that the emitting region is endowed by a magnetic field, electrons and protons are cooled down by electromagnetic (synchrotron radiation and Compton scattering) and hadronic (proton-photon interaction) channels, respectively. Taking into account the cooling processes for electron and proton distributions, the observed spectra for electron synchrotron radiation, Compton scattering and

$\dagger$ Luc Binette-Fundación UNAM Fellow. 


\begin{tabular}{|c|c|c|c|}
\hline Parameter & Value & Parameter & Value \\
\hline$A_{\operatorname{syn}, \gamma}\left(\mathrm{MeVcm}^{-2} \mathrm{~s}^{-1}\right)$ & $(6.248 \pm 0.899) \times 10^{-5}$ & $A_{s s c, \gamma}\left(\mathrm{MeVcm}^{-2} \mathrm{~s}^{-1}\right)$ & $(1.806 \pm 0.754) \times 10^{-5}$ \\
\hline$\alpha_{e}$ & $2.809 \pm 0.0520$ & $\alpha_{e}$ & $2.809 \pm 0.0520$ \\
\hline$\epsilon_{\gamma, c}^{s y n}(\mathrm{eV})$ & $0.100 \pm 0.001$ & $\epsilon_{\gamma, c}^{s s c}(\mathrm{MeV})$ & $7.254 \pm 2.574$ \\
\hline$\epsilon_{\gamma, m}^{s y n}(\mathrm{eV})$ & $(0.001 \pm 9.308) \times 10^{-05}$ & $\epsilon_{\gamma, m}^{s s c}(\mathrm{keV})$ & $(11.374 \pm 3.696)$ \\
\hline
\end{tabular}

Table 1. Best fit electron synchrotron radiation (left) and Compton scattering (right) parameters obtained after fitting the SED.

proton-photon interaction are (Fraija 2014a, Fraija et al. 2012):

$$
\begin{gathered}
\left(\epsilon^{2} N(\epsilon)\right)_{\gamma, s y n}=A_{s y n, \gamma} \begin{cases}\left(\frac{\epsilon_{\gamma}}{\epsilon_{\gamma, m}^{s y n}}\right)^{4 / 3} & \epsilon_{\gamma}<\epsilon_{\gamma, m}^{s y n} \\
\left(\frac{\epsilon_{\gamma}}{\epsilon_{\gamma, y}^{s y n}}\right)^{-(\alpha-3) / 2} & \epsilon_{\gamma, m}^{s y n}<\epsilon_{\gamma}<\epsilon_{\gamma, c}^{s y n} \\
\left(\frac{\epsilon_{\gamma, c}^{\prime, c}}{\epsilon_{\gamma, m}^{s, n}}\right)^{-(\alpha-3) / 2}\left(\frac{\epsilon_{\gamma}}{\epsilon_{\gamma, c}^{s y n}}\right)^{-(\alpha-2) / 2}, & \epsilon_{\gamma, c}^{s y n}<\epsilon_{\gamma}<\epsilon_{\gamma, m a x}^{s y n},\end{cases} \\
\left(\epsilon^{2} N(\epsilon)\right)_{\gamma, s s c}=A_{s s c, \gamma} \begin{cases}\left(\frac{\epsilon_{\gamma}}{\epsilon_{\gamma, m}^{s s c}}\right)^{4 / 3} & \epsilon_{\gamma}<\epsilon_{\gamma, m}^{s s c}, \\
\left(\frac{\epsilon_{\gamma}}{\epsilon_{\gamma, m}^{s s c}}\right)^{-(\alpha-3) / 2} & \epsilon_{\gamma, m}^{s s c}<\epsilon_{\gamma}<\epsilon_{\gamma, c}^{s s c}, \\
\left(\frac{\epsilon_{\gamma, c}^{s s c}}{\epsilon_{\gamma, m}^{s s c}}\right)^{-(\alpha-3) / 2}\left(\frac{\epsilon_{\gamma}}{\epsilon_{\gamma, c}^{s c}}\right)^{-(\alpha-2) / 2} & \epsilon_{\gamma, c}^{s s c}<\epsilon_{\gamma}<\epsilon_{\gamma, m a x}^{s s c},\end{cases} \\
\left(\epsilon^{2} N(\epsilon)\right)_{\pi^{0}, \gamma}=A_{p, \gamma} \begin{cases}\left(\frac{\epsilon_{\gamma}}{\epsilon_{0}}\right)^{-1}\left(\frac{\epsilon_{\gamma, c, \pi}}{\epsilon_{0}}\right)^{-\alpha_{p}+3} & \epsilon_{\gamma}<\epsilon_{\gamma, c, \pi} \\
\left(\frac{\epsilon_{\gamma}}{\epsilon_{0}}\right)^{-\alpha_{p}+2} & \epsilon_{\gamma, c, \pi}<\epsilon_{\gamma},\end{cases}
\end{gathered}
$$

respectively, where $A_{s y n, \gamma}\left(A_{s s c, \gamma}\right), \epsilon_{\gamma, m}^{s y n}\left(\epsilon_{\gamma, m}^{s s c}\right), \epsilon_{\gamma, c}^{s y n}\left(\epsilon_{\gamma, c}^{s s c}\right)$ and $\epsilon_{\gamma, \text { max }}^{s y n}\left(\epsilon_{\gamma, \text { max }}^{s s c}\right)$ are the proportionality constant and break energies for characteristic, cut-off and maximum of electron synchrotron (Compton scattering) spectrum and $A_{p, \gamma}$ and $\epsilon_{\gamma, c, \pi^{0}}$ are the proportionality constant and break photon energy for photo-pion spectrum (Fraija 2014b). It is important to highlight that the neutrino counterpart is calculated through $\int \frac{d N_{\nu}}{d E_{\nu}} E_{\nu} d E_{\nu}=\frac{1}{4} \int\left(\frac{d N}{d \epsilon}\right)_{\pi^{0}, \gamma} \epsilon_{\pi^{0}, \gamma} d \epsilon_{\pi^{0}, \gamma}$, where the neutrino flux is $d N_{\nu} / d E_{\nu}=A_{\nu} E_{\nu}^{-\alpha_{\nu}}$, with $\alpha_{\nu} \simeq \alpha_{p}$.

\section{Results}

We have presented a lepton-hadronic model through Eqns. 2.2, 2.2 and 2.3 to describe the broadband SED of NGC1275. For the hadronic interactions we invoke the p $\gamma$ interactions of Fermi-accelerated protons with the target photons at the second SSC peak. To find the best fit of our leptonic and hadronic models, we use the chi-square $\left(\chi^{2}\right)$ minimization method. The fit values for the leptonic model are shown in Table 1, while for hadronic model are $A_{p \gamma}=(2.932 \pm 0.6579) \times 10^{-6} \mathrm{MeV} \mathrm{cm}^{-2} \mathrm{~s}^{-1}$ and $\alpha_{p}=3.467 \pm 0.090$. The leptonic model describes the photon spectrum up to a few GeVs while the hadronic model up to hundreds of GeVs. The neutrino event expected in a $\mathrm{km}^{3}$ Cherenkov telescope is $0.93 \times 10^{-4}$ per year.

\section{References}

Abdo, A. A., et al. 2009, ApJ, 699, 31

Acciari, V. A., et al. 2009, ApJ, 706, 275

Aleksić, J., et al. 2012, A\& $A, 539$, L2

Fraija, N., et al. 2012, ApJ, 753, 40

Fraija, N. 2014a, MNRAS, 441, 1209

Fraija, N. 2014b, ApJ, 783, 44

Veron, P. 1978, Nature, 272, 430 